\title{
Surgical Management of Lumbar Neurofibroma
}

\author{
AHMED A. MOHAMED, M.D.* and MOHAMMED F. ADEL ALI, M.D.** \\ The Department of Neurosurgery, Faculties of Medicine, Bani Swef* and Cairo** Universities
}

\begin{abstract}
Background: Neurofibroma is a benign nerve sheath tumor in the peripheral nervous system. In $90 \%$ of cases they're found as stand-alone tumors, while the remainder are found with neurofibromatosis type I (NF1). Lumbar neurofibroma often encountered in the surgical practice, presenting with sciatic pain, tingling and numbness, or even foot drop.
\end{abstract}

Aim of Study: The aim of this study is to evaluate the role of surgery and defining the prognostic factors that affect the outcome of microscopic resection and how to avoid the possible complications especially those related to spinal instability and CSF leak.

Patients and Methods: This is a retrospective study of sixteen cases of lumbar neurofibroma operated in the departments of the authors by gross total resection, the surgery was microscopic assisted to secure the lumbar roots, and improve the outcome.

Results: In this study, the male-to-female ratio of patients was 3 to $1(75 \%$ to $25 \%)$. The mean age at the time of operation was 29 years. The initial symptoms were pain whether back or leg pain, gait disturbance resulting from leg motor weakness in $12 \%$ of our patients, tingling and numbness was found in 10 patients. Total removal of the tumor was achieved in 14 cases. Post-operative complications included CSFpseudo meningocele in onecase $(6 \%)$, wound infection (including superficial and deep) in 3 cases (19\%). One patient did not experience an improvement of the presenting motor power deficit.

Conclusion: Excision of the lumbar neurofibroma totally can be achieved safely without complication if proper microscopic surgery principles were followed with special concerns directed towards the dissection of the tumor from adherent nerve roots especially the hidden ones. Midline posterior approach is a convenient approach that can guarantee spinal stability. Extreme attention should be applied towards closure steps of the surgery especially those related to the dural seal.

Key Words: Neurofibroma Type 1 (NF1) - Spinal Nerve Sheath Tumors (NSTs).

Correspondence to: Dr. Ahmed A. Mohamed, The Department of Neurosurgery, Faculty of Medicine, Bani Swef University

\section{Introduction}

SPINAL Nerve Sheath Tumors (NSTs) are consistent pathologically with schwannomas or neurofibromas. They are common types of spinal cord tumors and may constitute up to $33 \%$ of intradural spinal tumors [1-3]. They occur both sporadically and in association with neurofibromatosis 1 (NF1; von Recklinghausen's disease) which is one of the most common autosomal dominant disorders, affecting 1 in 3500 individuals, with significant heterogeneity in its clinical manifestation [4-7].

Lumbar neurofibromas can present by diversity of nonspecific clinical manifestations, which makes its diagnosis challenging especially when the symptoms of the patient are vague like low back pain, leg pain and gait disturbance. More severe symptoms can be encountered like urinary dysfunction when spinal NSTs originate around the TLJ. Then the condition may mimic epiconus, conus, or cauda equina syndrome $[\mathbf{8 , 9}$. When the results of neuroimaging studies come back, the news may be devastating for the patients as usually they are psychologically prepared for having a usual degenerative spine condition, nevertheless, those tumors are basically benign in nature with slow growth and have a low rate of local invasion or distant metastasis. Moreover, these tumors can be addressed surgically with high levels of success.

The stumbling blocks which face this good outcome are both, post-operative complications and the slow or even absence of the recovery of the pre-operative neurologic deficits which may be related to the delay of correct diagnosis. In this study, we will present our experience to handle lumbar neurofibromas with a way to magnify their discovery among patients in the outpatient clinics, and also we will illustrate our strategies tominimize the post-operative complications that set back the recovery journey. 


\section{Material and Methods}

The clinical records of patients with pathologicalconfirmation with lumbarneurofibroma atthe author's institutes, between January 2015 and August 2018 were retrospectively reviewed. The clinical characteristics, radiological, and pathological data were collected with focusing on the age of the patient, gender, presenting symptoms, family history, presence of other signs of neurofibromatosis Type 1 or Type 2, tumor site, size of tumor (in preoperative MRI), extent of resection (by MRI post-operatively or operative report), post-operative complications, treatment outcome, time to last follow-up and follow-up imaging.

Patients with a single spinal neurofibroma and without other stigmata of NF 1 or NF2 were classified as patients with solitary neurofibroma. Patients who fulfilled the criteria for diagnosis of NF 1 or had constitutional NF 1 mutations were diagnosed with NF 1.

\section{Surgical technique:}

After induction of anesthesia, a prone position is used. Fluoroscopic guidance is used to determine the level of the tumor both before skin incision and after muscle separation and exposure of the laminae which is a very crucial step in order not to expand the bony work beyond required which furtherly avoids the possible spinal instability.

Through a posterior midline approach, the paravertebral muscles are detached from the spinous processes and laminae. The laminae are then cut carefully just medial to the facet joints on both sides. We used to finish the bony work without jeopardizing the facet joints bilaterally which protect against spinal instability later on. With the exposure of the dura, a snip is then made within it to allow a slow release of CSF. After widening of the dural incision, take up sutures are obtained to maintain the dural opening. Cottonoid pads are then applied over the roots to protect them during the suction of CSF.

Arachnoid dissection then is done using a sharp knife. In small tumors, one can identify the root from which the tumor arises and can apply coagulation via a low current bipolar to seal the blood vessels within the root which is then cut by micro scissors at the upper and the lower poles of the tumor. If the tumor is large, then internal debulking is carried out until it is possible to mobilize the walls of the tumor to gain access around it and undergo the previous steps. All over the operation use a cottonoid pad under the suction. In many cases the surgeon can encounter a more anterior normal motor root which is obscured by the tumor and adherent to its wall. It can be damaged easily unless careful sharp dissection is made. We strongly advocate the use of cottonoid pads whenever suction is needed to prevent unintended damage of the rootlets by the suction power even if minimal. After the tumor is completely exercised, irrigation with saline is applied to wash the blood from the tumor bed followed by closure of the dura in a watertight fashion, fibrin glue can be put over the stitch to face CSF leak. The anesthesia physician is then asked to perform a valsalva maneuver to make sure that there is no leakage through the dural repair. Then closure of the fascia is carried on aalso in watertight fashion after putting off a drain not under suction. This subfascialdrain will be removed after two days followed by suturing of its orifice.

After the operation, an external brace is used for four weeks after surgery. Patients begin to ambulate with the brace one day after surgery.

\section{Surgical evaluation and follow-up:}

These patients were followed-up through telephone interviews or an outpatient clinic visit to obtain more detailed medical information.

During post-operative follow-up, an X-ray of the lumbar area was done for all patients to assess the alignment of spine, and measure the Cobb angle (the angle between intersecting lines drawn perpendicular to the top of the vertebra one-level above the top vertebra, and the bottom of the vertebra one-level below the bottom vertebra) and compared topreoperative X-ray to evaluate sagittal alignment of the spine. MRI was used to observe the epidural scar and exclude tumor recurrence.

\section{Results}

\section{Demographics:}

Between January 2015 and August 2018, 16 patients underwent surgery for lumbar neurofibroma at our institutions. The mean age at the time of operation was 29 years. There was a male predominance with 12 tumors $(75 \%)$ in men and 4 tumors $(25 \%)$ in women.

\section{Clinical manifestations:}

The most common initial symptom was pain which varied between leg pain in 9 patients $(56 \%)$, back pain in 6 patients $(31 \%)$. The second most 
common symptom was tingling and numbness which were present in 10 patients $(62 \%)$. The most critical symptom in our series was gait disturbance resulting from leg motor weakness and this was the condition in 2 patients (12\%).

\section{Surgical findings:}

Location of the tumor was intradural in 14 cases $(88 \%)$ extradural in 1 case $(6 \%)$, dumbbell in 1 cases $(6 \%)$.

The average operative time was $137 \mathrm{~min}$ (range 90-205min). Average estimated blood loss during surgery was $510 \mathrm{~mL}$ (range 350 to $670 \mathrm{~mL}$ ).

Most of the tumors in our series occupied 2 lumbar levels which was the case in 12 patients $(75 \%)$. In 3 patients, the tumor was large and occupied 3 levels of lumbar spine, while in one patient the tumor was limited to only one level. The extension of the tumor does affect the bony work required for full exposure of the tumor which facilitates its removal, so we did the laminectomies in a number that matched the tumor extension.

Total removal of the tumor was achieved in 14 cases $(87,5 \%)$. It was not complete in 2 cases $(12.5 \%)$; one with subpial growth and one with foraminal extension at initial surgery. The case with foraminal extension was partially resected, with a small part of the tumor being left in the neural foramen. The residual tumor in the distal neural foramen was quite stable during the followup period.

\section{Patients outcome:}

Among our series, 10 patients had solitary neurofibroma. (Rest of pathologies) the result of surgical intervention was recovery (no evident symptoms or signs) in 13 cases (81\%), 2 of them experienced moderate back pain in the first 2 months after surgery but eventually they recovered completely. One of the patients with pre-operative motor weakness improved from motor power grade 3 pre-operative to reach motor power grade 4+ post-operatively, the other patient did not improve. This may be attributed to the long period between the incident of weakness and surgical intervention to remove the offending lesion. One patient has persistent tingling and numbness in the lower limb yet there is no motor affection. CSFpseudo meningocele did happen in 1 case (6\%), wound infection (including superficial and deep) in 2 cases $(12.5 \%)$ which was treated by frequent dressing and did not need debridement. We did not encounter spinal deformity requiring surgical intervention in our series through the follow-up period despite the long segment laminectomies that we were forced to do in patients with large tumors.

\section{Follow-up:}

The post-operative evaluation was done periodically at the out-patient clinic. The average time of follow-up was 32.5 months (25-40 months).

\section{Illustrative case:}

A 43-year-old female patient presented with severe low back pain with the characteristic feature of increasing at bed time and also she had severe lower limb neuropathic pain (sciatic pain) which increased cough and straining. Both pains were Partiallyrelieved by rest and analgesic. Upon examination, we couldn't find the classic stretch signs and movement limitations that usually distiguish the degenerative lumbar spine conditions. Despite being free from neurologic deficits, we urged MRI lumbosacral spine which revealed L3-4 intradural mass Fig. (1). We approached the tumor via posterior midline approach with L3 and L4 laminectomies to expose the upper and lower poles of the tumor then we applied the aforementioned surgical strategies. The patient underwent an uneventful post-operative course with being intact neurologically and she reported relief of her back and lower limb pains. 3 months after the operation, she underwent an MRI follow-up which revealed satisfactory tumor excision with no evidence of recurrence Fig. (2).
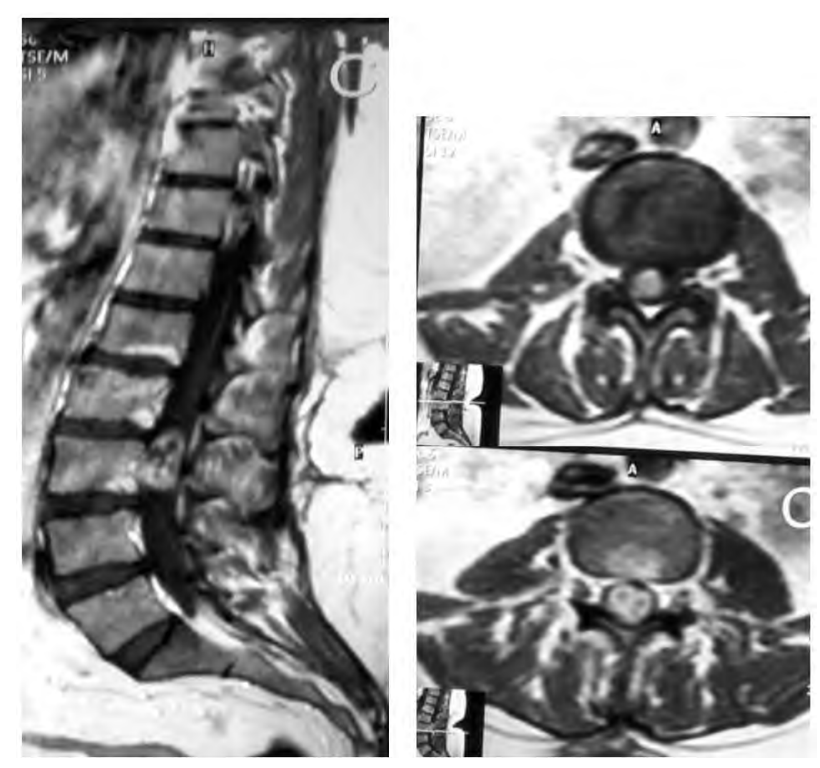

Fig. (1): Represents the pre-operative MRI study with contrast of a female patient with lumbar neurofibroma at the level of L3 and L4. 


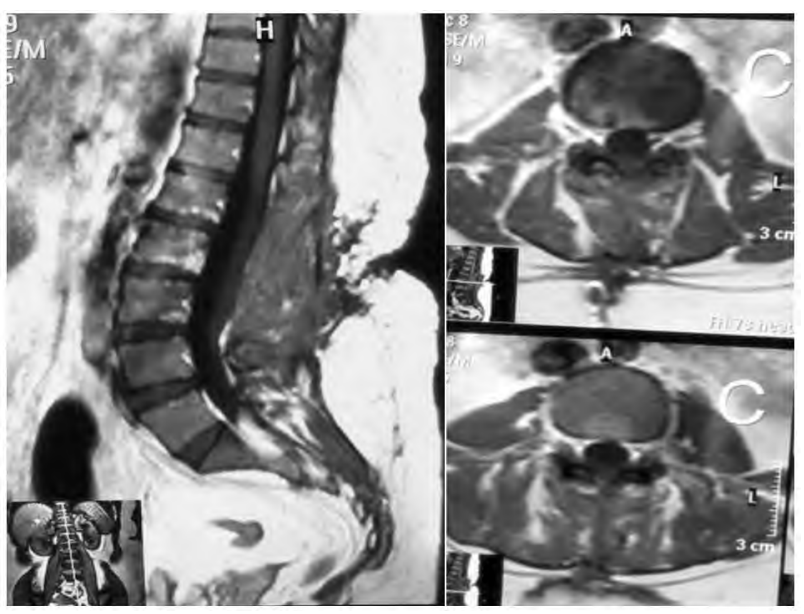

Fig. (2): Illustrating the post-operative MRI study with contrast of the patient with total excision of the lumbar neurofibroma at the level of L3 and L4 with no evidence of recurrence.

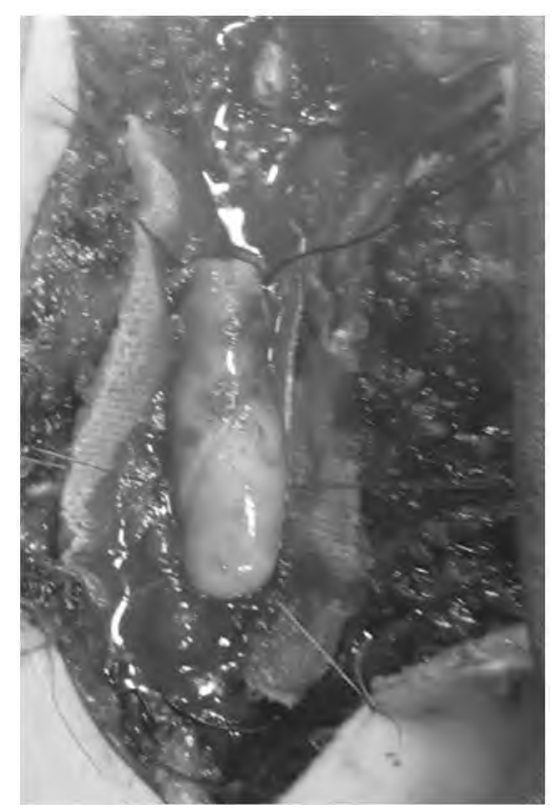

Fig. (3): Illustrates an intra-operative image of a lumbar neurofibroma operated via posterior midline approach and laminectomy.

\section{Discussion}

Lumbar nerve sheath tumors are very challenging in diagnosis. Having no particular symptoms $[10,14]$, they can easily be misdiagnosed as lumbar degenerative conditions. The chief symptom in our series was pain similar to other series $[2,20]$ which is really a very vague complaint and is subjective in nature. Moreover this pain can be ignored by the patient himself which delays the medical advice or can be misleading to the surgeon to the extent that he might think that this patient is exaggerating his symptoms to get sick leaves from work which is unfortunately a trend in many areas of the world especially in the setting of third world countries in which the problem is escalated by the poor access to the neuroimaging like MRI. The aforementioned factors significantly affect the timing of diagnosis of the lumbar nerve sheath tumors with development of more complex symptoms like motor and sensory deficits which contribute to worsening of the overall outcome of these tumors, despite the fact that the majority of spinal NSTs are benign in nature with a slow-growing pattern. A clinical correlation between poor neurological status before surgery and unfavorable outcomes has been previously described $[\mathbf{1 1}, \mathbf{1 4}, \mathbf{1 5}]$, although acute deterioration rarely happens because of torsion or hemorrhage [16,17]. Wostrack et al., analyzed surgical cases of conus medullaris and cauda equina tumors and suggested that the reversibility of preoperative symptoms is related to the duration between symptom onset and surgery and to the presence of pre-operative neurological deficits [10].

So, we advocated an algorithm of management of patients coming to our Outpatient Clinics (OPC) with back pain and/or leg pain by which we can pick up those patients early enough to be managed successfully with favorable outcomes. In the first visit to OPC, if the history of the back pain was not related to a trauma or faulty movement, then we ask for X-ray studies to the lumbar regions to delineate any degenerative condition that might be present and explains the patient symptoms after which we prescribe him medications accordingly for one week. In the second visit, if there was no improvement of the symptoms, we ask for an MRI to the lumbar region to detect the responsible pathology. This protocol helped us in detecting 7 patients in our series. Surprisingly one of them has the tumor extending to 3 levels of lumbar spine and causing only back pain. If the patient is presented with an already established neural deficit, we skip the step of X-ray and we ask for an MRI. Despite the economic challenges in our country, we try every effort to do these neuroimaging studies. In fact, there are a lot of charity facilities that help in providing such services without increasing the burdens in our institutes.

Treatment of neurofibromas can be particularly satisfying for the surgeon because of their benign nature and highly successful results of removal. However it is still a challenging procedure because the surgeon here isdealing with lumbar roots which can be easily injured even by suction.

In our series, successful surgical resection of lumbar neurofibroma was achieved using a posterior approach followed by laminectomies as long as the sagittal extension of the tumors. Some studies 
advocated a minimal invasive approach with very minimal or even absent bony work like Y.J. Zhu et al., yet their studies included lesions of much less sizes. We believe that preservation of the paraspinal muscles and facet joints are the key issues in the spine stability as mentioned in previous studies $[\mathbf{1 8 , 1 9 ]}$. Therefore, we did not have to apply fixation to any of our patients despite the long segment laminectomies that we were compelled to do in some patients to remove the tumors completely. The lumbar brace application to the back after surgery for a brief period can contribute to decrease the muscular strain and maintain spinal stability till the muscular tissue recovery. However, we advocate regular X-ray follow-up to detect any resultant lumbar instability especially in persistent back pain if ever happened.

Serious complications can result from surgical intervention to lumbar nerve sheath tumors. Surgical complications include wound infections, CSF leaks, Deep Venous Thrombosis (DVT), or pulmonary complications, new or worsening motor and sensory deficits could happen with rates ranging from $5 \%$ to $30 \%$ [2,11-13,22,23].

Kim et al., reported that among 31 patients who had undergone nerve sacrifice, 7 (22\%) developed partial loss of strength or sensation, though none was debilitating [20]. These authors concluded that nerve roots associated with spinal neurofibroma were often nonfunctional and that the incidence of developing disabling neurological deficits after nerve sacrifice was very minimal.we believe that their hypothesis is right, and we advocate the sacrifice of the root of origin of the tumor to guarantee proper radicality, nevertheless extreme caution should applied when mobilizing the deep surface of the tumor capsule to guard against the injury of the anteriorly located motor root which sometimes becomes adherent to the tumor capsule and necessitates sharp dissection.

CSF leak is a serious complication of surgical excision of these tumors. The condition may complicate to become a life-threatening matter if accompanied by infection and subsequently meningitis. We advocate a 4-tier approach to diminish the probabilities of the CSF leak which consists of: Watertight closure of the dura with absorbable sutures augmented by tissue glue application then Valsalva maneuver to delineate any possible leaking points followed by closure of the fascia in watertight fashion and at last, insertion of sub fascial drain without suction setting to be removed after 2 days with sealing of its orifice by already applied loose stitch to be tighten afterwards. It is quite an effective strategy that does not consume time (our mean skin to skin time was 137 minutes). It lowered our CSF leak complications down to only one case with pseudo meningiocele which were resolved with conservative management reflecting how much the leaking point was trivial.

This study is limited by its short follow-up period. The mean time from surgery to the last clinic visit was 32.5 months; therefore, our rates of non-improved neurological deficit may actually be overestimates since in some cases neurological function can improve over time. Also the study is limited by the small number of the cases because of the rarity of these lesions in the lumbar area. But we hope that future studies could address a greater number of patients with lumbar NST with longer follow-up periods to underscore preoperative characteristics or radiological features that may predict ease of resection or intraoperative difficulties.

\section{Conclusions:}

Excision of the lumbar neurofibroma totally can be achieved safely without complication if proper microscopic surgery principles were followed with special concerns directed towards the dissection of the tumor from adherent nerve roots especially the hidden ones. Midline posterior approach represents a golden gate to address such tumors without jeopardizing the spinal stability especially if the facets were maintained intact. Extreme attention should be applied towards closure steps of the surgery especially those related to the dural seal. We presented our 4-step protocol for proper closure of the dura and fascia aiming at standardization of these steps to diminish the overwhelming complications related to CSF leak.

\section{References}

1- ENGELHARD H.H., VILLANO J.L., PORTER K.R., STEWART A.K., BARUA M., BARKER F.G. and NEWTON H.B.: Clinical presentation, histology, and treatment in 430 patients with primary tumors of the spinal cord, spinal meninges, or cauda equina. J. Neurosurg. Spine, 13: 67-77, 2010.

2- SEPPÄLÄ M.T., HALTIA M.J., SANKILA R.J., JÄÄSKELÄINEN J.E. and HEIS-KANEN O.: Long-term outcome after removal of spinal schwan-noma: A clinicopathological study of 187 cases. J. Neurosurg., 83: 6216, 1995.

3- KLEKAMP J. and SAMII M.: Introduction of a score system for the clinical evaluation of patients with spinal processes. Acta Neurochir., 123: 221-3, 1993.

4- MARCHUK D.A., SAULINO A.M., TAVAKKOL R., et al.: cDNA cloning of the type 1 neurofibromatosis gene: Complete sequence of the NF1 gene product. Genomics, 11: 931-40, 1991. 
5- THAKKAR S.D., FEIGEN U. and MAUTNER V.F.: Spinal tumors in neurofibromatosis type 1: An MRI study of frequency, multiplicity and variety. Neuroradiology, 41: 625-9, 1999.

6- TONSGARD J.H., KWAK S.M., SHORT M.P. and DACHMAN A.H.: CT imaging in adults with neuro bromatosis-1: Frequent asymptomatic plexiform lesions. Neurology, 50: 1755-60, 1998.

7- WIMMER K., MÜHLBAUER M., ECKART M., CALLENS T., REHDER H., BIRKNER T., et al.: A patient severely affected by spinal neurofibromas carries a recurrent splice site mutation in the NF1 gene. Eur. J. Hum. Genet., 10: 334-8, 2002.

8- EBNER F.H., ROSER F., ACIOLY M.A., SCHOEBER W. and TATAGIBA M.: Intramedullary lesions of the conus medullaris: Differential diagnosis and surgical management. Neurosurg. Rev., 32: 287-300, 2009.

9- WAGER M., LAPIERRE F., BLANC J.L., LISTRAT A. and BATAILLE B.: Cauda equina tumors: A French multicenter retrospective review of 231 adult cases and review of the literature. Neurosurg. Rev., 23: 119-29, 2000.

10- WOSTRACK M., SHIBAN E., OBERMUELLER T., GEMPT J., MEYER B. and RINGEL F.: Conus medullaris and cauda equina tumors: Clinical presentation, prognosis, and outcome after surgical treatment. J. Neurosurg. Spine, 20: 335-43, 2014.

11- CONTI P., PANSINI G., MOUCHATY H., CAPUANO C. and CONTI R.: Spinal neurinomas: Retrospective analysis and long-term outcome of 179 consecutively operated cases and review of the literature. Surg. Neurol., 61: 34-43, 2004.

12- FERNANDES R.L., LYNCH J.C., WELLING L., GONÇALVES M., TRAGANTE R., TEMPONI V., et al.: Complete removal of the spinal nerve sheath tumors. Surgical technics and results from a series of 30 patients. Arq. Neuropsiquiatr., 72: 312-7, 2014.

13- CELLI P.: Treatment of relevant nerve roots involved in nerve sheath tumors: Removal or preservation? Neurosurgery, 51: 684-92, 2002.

14- HAN I.H., KUH S.U., CHIN D.K., KIM K.S., JIN B.H. and CHO Y.E.: Surgical treatment of primary spinal tumors in the conus medullaris. J. Korean Neurosurg. Soc., 44: 72-7, 2008.

15- FEARNSIDE M.R. and ADAMS C.B.: Tumours of the cauda equina. J. Neurol. Neurosurg. Psychiatry, 41: 24 31, 1978.

16- ICHINOSE T., TAKAMI T., YAMAMOTO N., TSUYUGUCHI N. and OHATA K.: Intratumoral hemorrhage of spinal schwannoma of the cauda equina manifesting as acute paraparesis-case report. Neurol. Med. Chir. (Tokyo), 49: 255-7, 2009.

17- JENKINS A.L. $3^{\text {rd }}$, AHUJA A., OLIFF A.H., SOBOTKA S.: Spinal schwannoma presenting due to torsion and hemorrhage: Case report and review of literature. Spine J., 15: e1-e4, 2015.

18- OKTEM I.S., AKDEMIR H., KURTSOY A., KOÇ R.K., MENKÜ A. and TUCER B.: Hemilaminectomy for the removal of the spinal lesions. Spinal Cord., 38: 92-6, 2000.

19- ZANDER T., ROHLMANN A., KLÖCKNER C. and BERGMANN G.: Influence of graded facetectomy and laminectomy on spinal biomechanics. Eur. Spine J., 12: 427-43, 2003.

20- KIM P., EBERSOLD M.J., ONOFRIO B.M. and QUAST L.M.: Surgery of spinal nerve schwannoma. Risk of neurological de cit after resection of involved root. J. Neurosurg., 71: 810-4, 1989.

21- SAFAVI-ABBASI S., SENOGLU M., THEODORE N., WORKMAN R.K., GHARABAGHI A., FEIZ-ERFAN I., et al.: Microsurgical management of spinal schwannomas: Evaluation of 128 cases. J. Neurosurg. Spinem 9: 40-7, 2008.

22- NANDA A., KUKREJA S., AMBEKAR S., BOLLAM P. and SIN A.H.: Surgical strategies in the management of spinal nerve sheath tumors. World Neurosurg., 83: 88699, 2015.

\section{التدخل الجراحى للآورام الليفية العصبية}

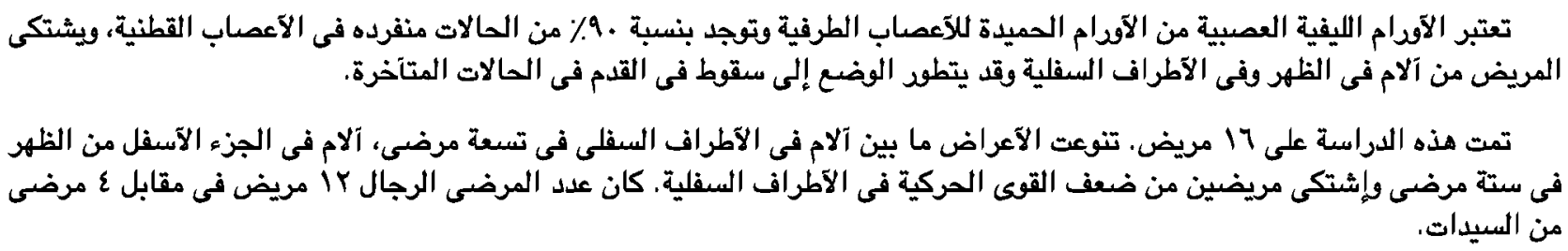

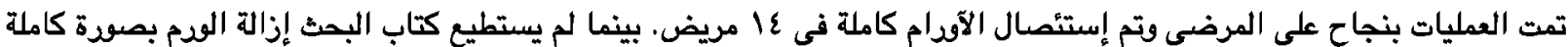

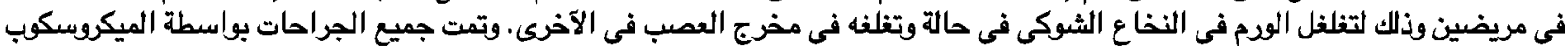
الجراحي.

نتصيح بإستئصال هذا النوع من الآقرام بطريقة آمنه عن طريق إستخدام الميكروبكوب الجراحى للحفاظ على جذور الآعصاب التى قد تكون آمام الورم وملتصقه بـ 